\title{
Prognostic Significance of Fibrotic Focus in Invasive Ductal Carcinoma of the Breast: A Prospective Observational Study
}

Takahiro Hasebe, M.D., Satoshi Sasaki, M.D., Shigeru Imoto, M.D., Kiyoshi Mukai, M.D., Tomoyuki Yokose, M.D., Atsushi Ochiai, M.D.

Pathology Division (TH, TY, AO); Epidemiology and Biostatistics Division (SS), National Cancer Center Research Institute East; Department of Surgery (SI), National Cancer Center Hospital East, Kashiwa, Chiba; and First Department of Pathology (KM), Tokyo Medical University, Tokyo, Japan

We have already reported invasive ductal carcinomas (IDCs) with fibrotic focus (FF) to be associated with significantly poorer survival than IDCs without FF. The purpose of this study was to prospectively investigate the effect of the presence of FF on the outcomes of 439 patients with IDCs to confirm the prognostic significance of FF, by the multivariate analysis, employing the Cox proportional hazard regression model, as compared with well-known clinicopathological parameters. We also precisely evaluated the prognostic significance of FF from the viewpoint of FF characteristics. The present study demonstrated that the presence of FF is a very useful parameter predicting tumor recurrence (TR), as well as initial distant organ metastasis (IDOM), in/lymph node-negative IDCs $(P=.024$ and $P=.026)$ and in IDCs positive for either or both estrogen receptor (ER) or progesterone receptor $(P R)(P=.007$ and $P=.015)$, respectively. In addition, FF of $>8 \mathbf{~ m m}$ in diameter was found to be an independent prognostic parameter for TR and IDOM in lymph node-negative patients and patients with IDC positive for either or both ER or $P R(P=.005$ and $P=.018)$. We conclude that the presence of FF is a very important histologic prognostic parameter for patients with IDCs of the breast.

KEY WORDS: Breast, Breast cancer, Fibrotic focus, Histology, Metastasis, Prognosis, Scar.

Mod Pathol 2002;15(5):502-516

Copyright ( $\odot 2002$ by The United States and Canadian Academy of Pathology, Inc.

VOL. 15, NO. 5, P. 502, 2002 Printed in the U.S.A.

Date of acceptance: February 4, 2002.

This work was supported in part by the following Grants-in-Aid from the Ministry of Health, Labor and Welfare (11-12), Japan: a for Cancer Research and a Grant-in-Aid for the Second Term Comprehensive 10-Year Strategy for Cancer Control.

Address reprint requests to: Atsushi Ochiai, M.D., Pathology Division, National Cancer Center Research Institute East, Kashiwanoha 6-5-1, Kashiwa, Chiba 277-8577 Japan; e-mail: aochiai@east.ncc.go.jp; fax: +81-471-34-6865.
Fibrotic focus (FF) is a mixture of fibroblasts and various amounts of collagen fibers and can occupy almost the entire center of an invasive ductal carcinoma (IDC). Breast cancer with FF-like lesions was previously described as a tumor with sclerotic foci by Linell et al. (1) and as a scar cancer by Fisher et al. (2). Scar cancers have been shown to have a better outcome and to be richer in steroid hormone receptors than nonscar cancers $(3,4)$. However, these studies on scar cancers did not classify cases according to histologic subtype and therefore included other histologic types, such as lobular, mucinous, medullary, or squamous cell carcinoma and noninvasive ductal carcinoma, in addition to invasive ductal carcinoma (IDG; 1-4). To more precisely clarify the clinicopathologic significance of FF in primary breast cancer, it is necessary to study tumors of the same histologic type.

We have already reported that IDCs with FF have more aggressive characteristics than do IDCs without FF (5), and the former are associated with significantly poorer short- and long-term survival periods than the latter $(6,7)$. We also found that IDCs with FF exhibit significantly greater tumor angiogenesis and higher tumor cell proliferative activity and that the presence of FF is an independent prognostic parameter for IDC patients (8). Recently, we clearly demonstrated that the highly proliferative fibroblasts forming FF play an important role in tumor progression of IDCs of the breast (9). In addition, Colpaert et al. (10) reported the prognostic significance of the presence of fibrotic focus in node-negative IDCs. Tsuda et al. $(11,12)$ reported that IDCs with a large central acellular zone, which are certainly a type of IDC with FF, also show poorer outcomes than those without such an acellular zone. These findings indicate that the presence of FF in IDCs is a very important histologic parameter for predicting outcome in patients with IDC of the breast. 
The purpose of this study was to prospectively investigate the effect of the presence of FF on the outcomes of patients with IDCs, as a means of confirming the prognostic significance of FF as evaluated in previous studies (5-7). In addition, we precisely evaluate the prognostic significance of FF from the viewpoint of FF characteristics. This study demonstrated that the presence of FF is a very useful parameter for predicting tumor recurrence and initial distant organ metastasis, respectively, in lymph node-negative IDCs and in IDCs positive for either or both estrogen receptor (ER) or progesterone receptor (PR). In addition, the combined assessment of the presence of FF and FF dimension was found to be an independent prognostic parameter for lymph node-negative and either or both ER or PR-positive IDC patients.

\section{MATERIALS AND METHODS}

\section{Cases}

Four hundred thirty-nine consecutive cases of IDC of the breast that were surgically treated between January 1993 and June 1999 at the National Cancer Center Hospital East constituted the basis of this study. Clinical information was obtained from the patients' medical records. All of the patients were Japanese women ranging in age from 28 to 78 years (median, $52 \mathrm{y}$ ), and all had a solitary lesion. There were 232 premenopausal patients and 207 postmenopausal patients. Standard radical mastectomy was done in 16 , partial mastectomy in 78 , and modified radical mastectomy in 345 women. Axillary lymph node dissection was carried out in all cases. None of the patients had received radiotherapy or chemotherapy before surgery, and 324 received adjuvant therapy. ERs and PRs in the cytosol fractions were determined by enzyme immunoassay (Otsuka Assay Laboratory, Tokushima, Japan). The upper cutoff values of the ER and PR assay are 13 and $10 \mathrm{fmol} / \mathrm{mg}$ of protein, respectively. All tumors were classified according to the pathological TNM (pTNM) classification (13).

For pathological examination, the surgically resected specimens were fixed in $10 \%$ formalin overnight at $4^{\circ} \mathrm{C}$, and the entire tumor was cut into slices at intervals of 0.5 to $0.7 \mathrm{~cm}$. The size and gross appearance of the tumors were recorded, and the former was validated by comparison with tumor size on histologic slides. Multiple histologic sections were taken from each tumor in order to measure the maximum tumor diameter and area. The sections were processed routinely and embedded in paraffin.

\section{Histological Examination}

Serial sections of each tumor area were cut from the paraffin blocks. One section was stained with hematoxylin and eosin and examined pathologically to confirm the diagnosis, and elastica staining was also performed to assess vascular invasion in all cases. The following histological parameters were evaluated in each IDC about 7 to 10 days after the operations: (1) invasive tumor size, (2) histologic grade, (3) tumor necrosis, (4) lymphatic invasion, (5) vascular invasion, (6) adipose tissue invasion, (7) skin invasion, and (8) muscle invasion. All tumors were classified according to the guidelines of the World Health Organization (14), and their histologic grade was evaluated based the Elston classification (15).

\section{Histological Features of FF and Their Assessment}

The presence of FF, and the following FF characteristics in each IDC, were also prospectively evaluated with the above eight histological parameters at the time of routine examination of the IDCs. FF is defined according to the following characteristics (5-9): (1) FF often consists of fibrous bands radially expanding into the surrounding area and have the appearance of a scar (Figs. 1A and 2C) or a radiating fibrosclerotic core (Fig. 2A). Some FFs have an irregular moth-eaten appearance (Fig. 3A). FFs are located at almost the exact center of the tumor. They are surrounded by a highly cellular zone of infiltrating ductal carcinoma cells and occupy variable percentages of the tumor area (Figs. 1A; 2, A and C; 3A; 4A). (2) Tumor cells are seldom seen in FFs of $\leq 3 \mathrm{~mm}$ in size (Fig. $4 \mathrm{~A}, \mathrm{C}$ ). However, tumor cells growing in a scirrhous fashion or in solid nests are observed in FFs as their size increases (Figs. 1B, 2B, 3B).3) The fibroblasts and collagen fibers in FFs are arranged in irregular or storiform patterns with increased fibroblasts cellularity and/or collagenization (Figs. 1B; 2B; 3B; 4, B and D). The degree of fibrosis in an FF is classified into three grades according to the following criteria: (1) Grade 1, a large number of fibroblasts with a small amount of collagen fibers; (2) Grade 3, mainly composed of collagen fibers, mostly hyalinized; and (3) Grade 2, intermediate between Grade 1 and 3, with fibroblasts and collagen fibers intermingled in various ratios (Figs. 1C, 1D, 2B, 2D, 3C, 4B and 4D). The arrangement of fibroblasts or collagen fibers forming FFs differs from that of the surrounding tumor stroma, which is more orderly, and from that of the normal breast tissue stroma. The difference in fibroblast arrangement is the characteristic that most distinguishes fibroblasts forming FFs from those outside FFs. Some FFs are associated with coagulation necrosis of the tumor. The area of tumor cell 

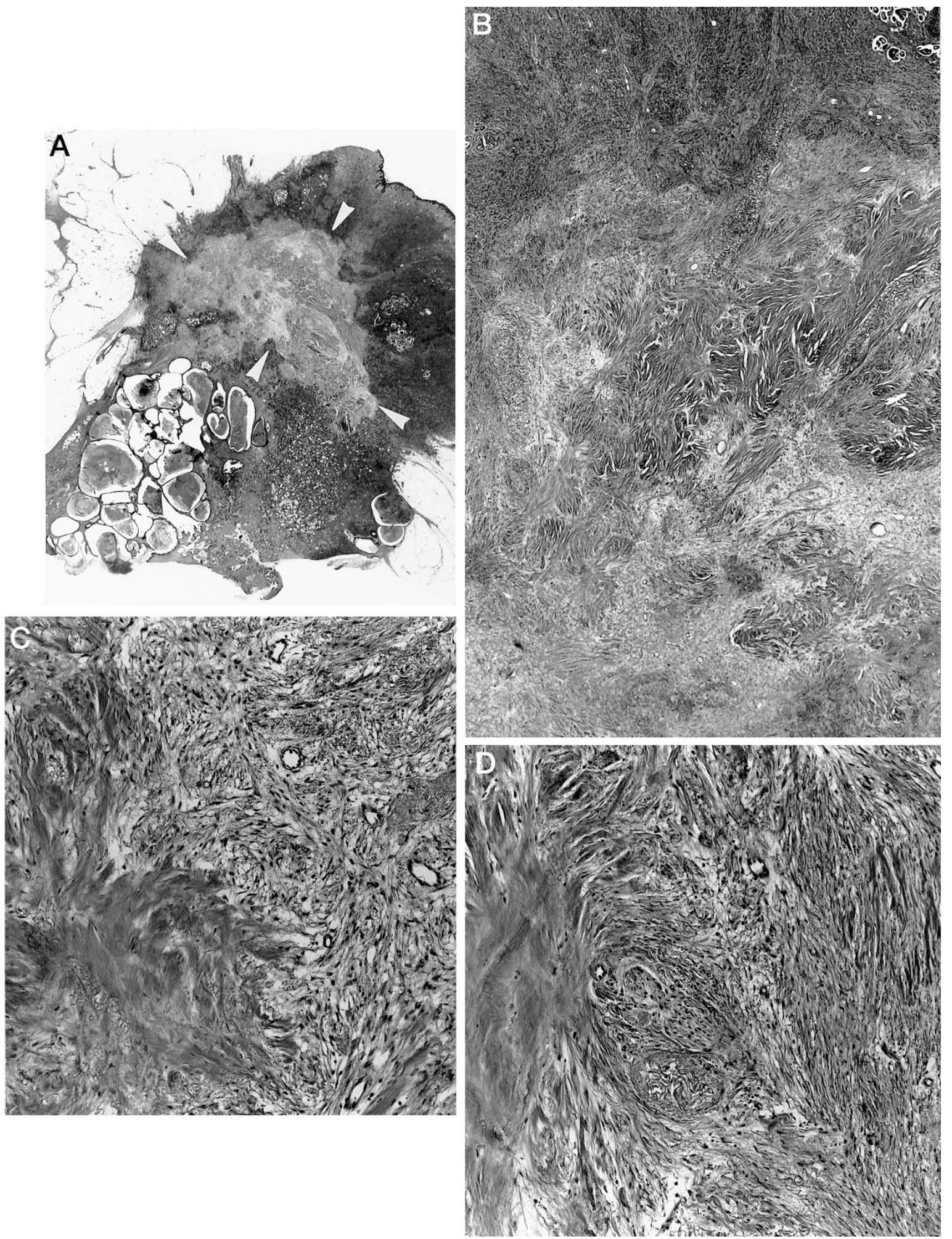

FIGURE 1. Invasive ductal carcinoma with fibrotic focus (FF). A, an FF measuring $10 \times 8 \mathrm{~mm}$ is observed within the tumor (arrowheads). The FF shows a scar-like feature and is surrounded by invasive ductal carcinoma cells. Some residual tumor islands of various sizes are observed within the FF (panoramic view). B, the fibroblasts and collagen fibers composing the FF show a storiform arrangement. C, the FF area consists of fibroblasts arranged in a storiform pattern (fibrosis Grade 1, right half), or hyalinized collagen fibers showing a storiform pattern (fibrosis Grade 3, left half). D, in other areas, the FF consists of fibroblasts and hyalinized collagen fibers showing a storiform arrangement (fibrosis Grade 2, right side to central portion), or only hyalinized collagen fibers showing a storiform pattern (fibrosis Grade 3, left one third).

coagulation necrosis within FF is smaller than that occupied by the fibroblasts or collagen fibers. Coagulation necrosis within tumors without accom- panying fibroblasts or collagen fiber proliferation is insufficient for a designation of FF. Whenever several FFs of various sizes were present in the same 


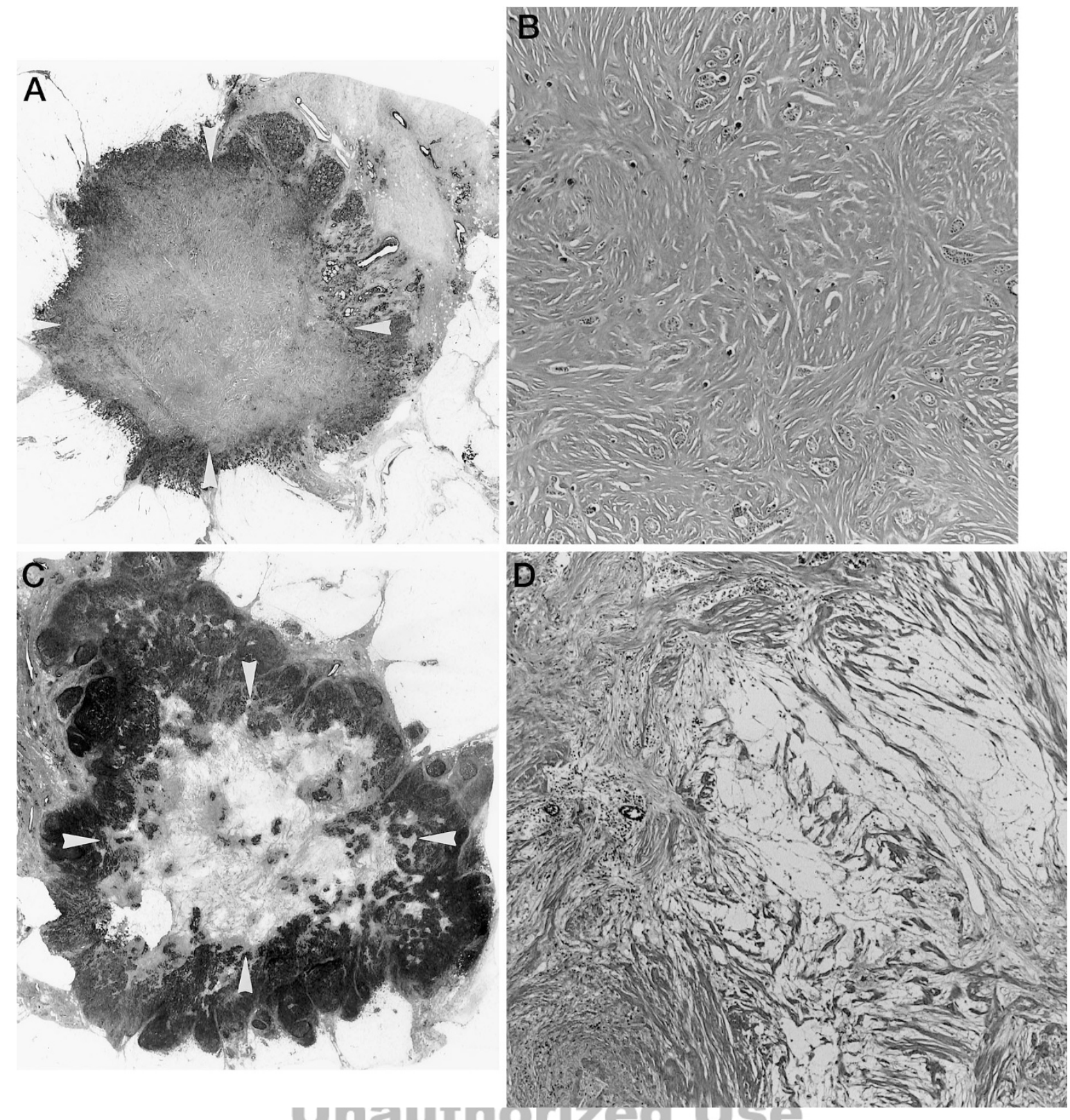

FIGURE 2. Invasive ductal carcinomas with fibrotic focus (FF). A, an FF measuring $11 \times 10 \mathrm{~mm}$ is observed within the tumor (arrowheads). The FF has the appearance of a radiating fibrosclerotic core (panoramic view). B, the FF consists of hyalinized collagen fibers admixed with calcium depositions (fibrosis Grade 3). The hyalinized collagen fibers show a storiform pattern. C, an FF measuring $15 \times 15$ mm is observed within the tumor (arrowheads). The FF shows a scar-like feature with residual tumor islands. D, the FF consists of fibroblasts admixed with collagen fibers (fibrosis Grade 2, left half), and loose-edematous matrices blending with small amounts of collagen fibers (right half).

tumor (Fig. 4A), the characteristics of the largest one were used for this study. In addition, a diameter of $\geq 1 \mathrm{~mm}$ is required to refer to a fibrosclerotic core as FF in IDC. The maximum dimension of FF in each IDC was measured with a microscope equipped with a $10 \times$ eyepiece enclosing a graticule consisting of a 9-vertical, 9-horizontal grid lattice that intersected at 81 points in each field. Most measurements were made with a $2 \times$ or $4 \times$ objective.

One author $(\mathrm{TH})$ routinely examined all of the histologic parameters and characteristics of FF in IDCs, and two other authors (KM or TY) immedi- ately identified the characteristics of all the IDCs to confirm the IDC characteristics recorded by TH. Whenever there was a discrepancy, both of the latter authors re-examined the slides to reach a consensus.

\section{Outcomes}

Patient survival was evaluated by follow-up for a median period of 50 months (range, 19 to $88 \mathrm{mo}$ ), to November of 2000. Three hundred thirty-eight patients were alive and well, 101 had tumor recurrence, 63 had initial distant organ metastasis, and 43 

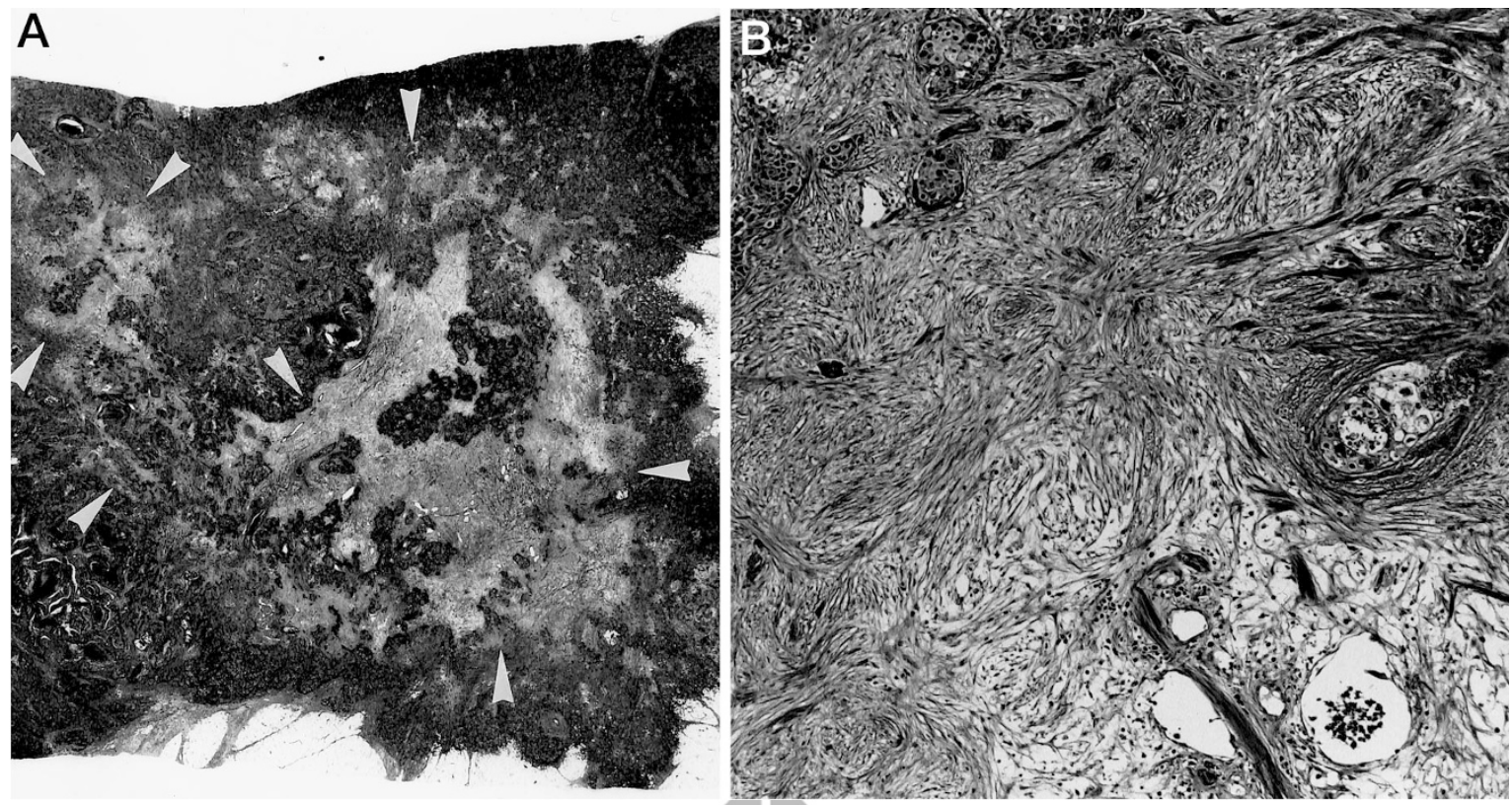

FIGURE 3. Invasive ductal carcinomas with fibrotic focus (FF). A, an FF measuring $15 \times 17 \mathrm{~mm}$ is observed within the tumor (arrowheads). The FF shows a moss-eaten-appearance and is surrounded by invasive ductal carcinoma cells. Some residual tumor islands of various sizes are observed within the FF (panoramic view). B, the fibroblasts and collagen fibers composing the FF show a storiform arrangement. The FF area consists of fibroblasts admixed with hyalinized collagen fibers.

had died of their disease. Measurements of diseasefree survival, initial distant organ metastasis-free survival, and overall survival started at the time of surgery. Tumor relapse was considered to have occurred whenever there was evidence of metastasis or local recurrence. Initial distant organ metastasis was observed in the following organs: (1) bone, 34 cases; (2) liver, 17 cases; (3) lung, 17 cases; and (4) brain, 4 cases. Only deaths caused by breast cancer were considered for the purpose of this study, and all patients who died of the disease had distant organ metastasis.

\section{Statistical Analysis}

We analyzed the parameters that were significantly associated with the presence of FF by the $\chi^{2}$ test. The following parameters were examined as potential predictors of the presence of FF: (1) age ( $\leq 39$ versus $>39$ ), (2) ER or PR status (either or both positive versus both negative), (3) invasive tumor size ( $\leq 20$ versus $>20 \mathrm{~mm}$ ), (4) histologic grade (Grade 1 or 2 versus Grade 3), (5) tumor necrosis (absent versus present), (6) lymphatic invasion (absent versus present), (7) vascular invasion (absent versus present), (8) adipose tissue invasion (absent versus present), (9) skin invasion (absent versus present), (10) nodal status (absent versus present), and (11) adjuvant therapy (no versus yes). Among these parameters, those significantly associated with the presence of FF in univariate analyses were entered into logistic regression multivariate analysis (16) using the step-down method until all remaining factors were significant at a $P$ value of
$<.05$, to identify the parameters most significantly associated with the presence of FF.

We prospectively analyzed the predictive power of the presence of FF for tumor recurrence, initial distant organ metastasis, and tumor death in the following groups: (1) all cases, (2) lymph node-negative and -positive cases, 3) cases positive for either or both ER or PR and cases negative for both, and (4) lymph node-negative cases and cases positive for either or both ER or PR by multivariate analysis employing the Cox proportional hazard regression model (17) with the step-down method until all remaining factors were significant at a $P$ value of $<.05$. In lymph nodenegative IDCs, and IDCs positive for either or both ER or PR, because $<10$ patients died of disease, we could only perform univariate analyses for tumor death. In addition, we could not perform multivariate analyses for tumor recurrence and initial distant organ metastasis in patients with lymph node-negative IDCs and patients positive for either or both ER or PR, because no patients died of the disease in this group. To conduct a comparative study for the presence of FF and tumor recurrence, initial distant organ metastasis, and tumor death, the parameters that had been evaluated by correlation with the presence of FF were again examined as potential predictors of tumor recurrence, initial distant organ metastasis, and tumor death. The parameters showing a significant correlation with tumor recurrence, initial distant organ metastasis, and tumor death in the univariate analyses using the log-rank test were then entered into 

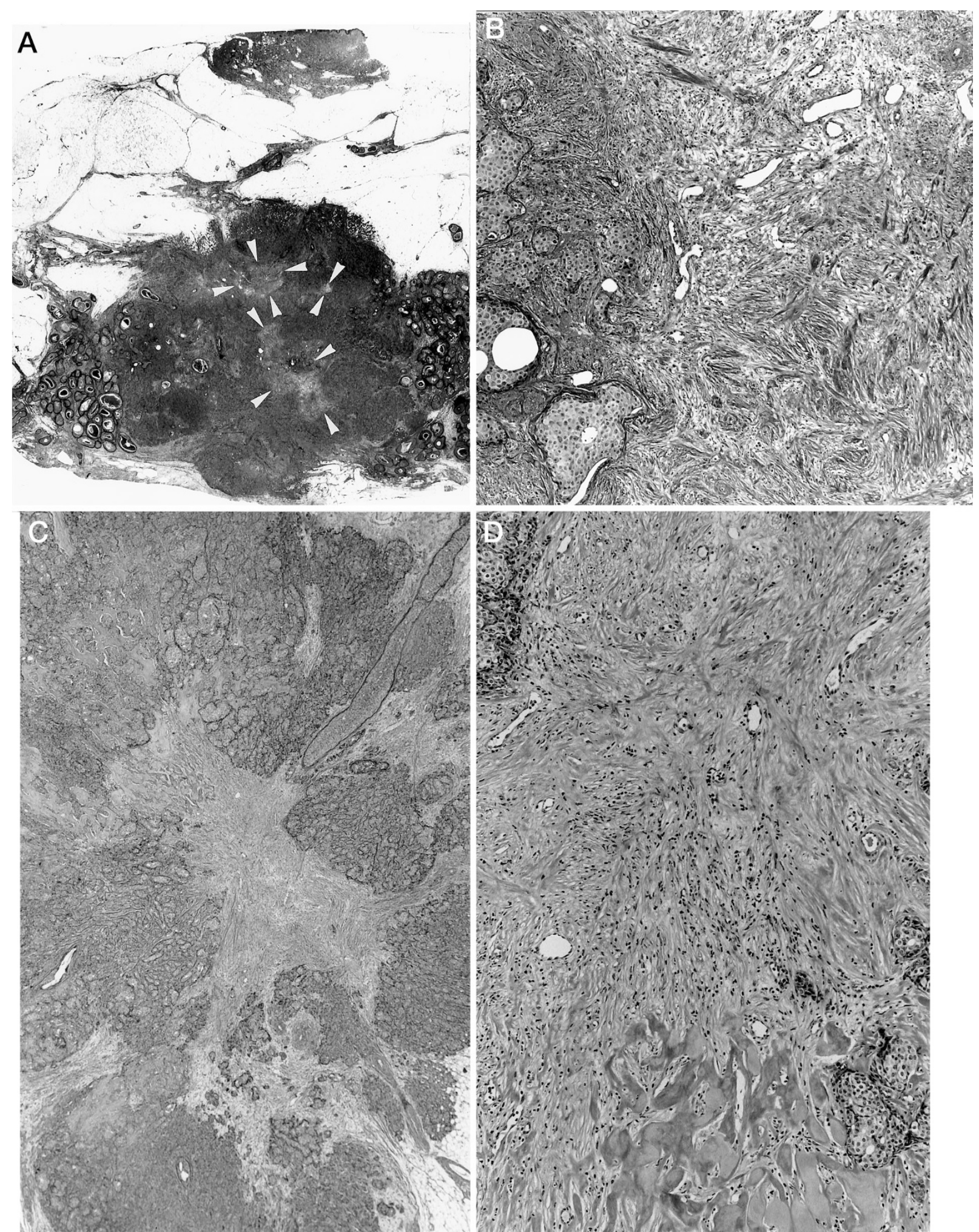

FIGURE 4. Invasive ductal carcinomas with fibrotic focus (FF). A, three FFs, the maximum size of which is $10 \times 7.2 \mathrm{~mm}$, are observed within the tumor (arrowheads). The smallest FF (indicated by two arrowheads) is $1.2 \times 1.0 \mathrm{~mm}$. B, the fibroblasts forming the FF are arranged in a storiform pattern and are intermingled with collagen fibers. C, a small FF measuring $4.0 \times 2.0 \mathrm{~mm}$ is observed within the tumor. $\mathbf{D}$, the FF area consists of fibroblasts admixed with collagen fibers, and hyalinized collagen fibers.

multivariate analyses. The survival curves for these parameters were drawn by the KaplanMeier method (18).
Next, we conducted on advanced analysis that evaluated the prognostic significance of the characteristics of FF in patients with IDCs with FF. For cases with 
FF $(n=235)$, the FF characteristics examined were the following: (1) maximum dimension (in millimeters) and (2) fibrosis grade (1, 2, and 3 ). The numbers of cases with IDCs with FF of Fibrosis Grades 1, 2, and 3 were 13,102 , and 120 , respectively. We previously reported that the presence of tumor necrosis within FF is a significant prognostic parameter for patients with IDCs with FF $(6,7)$. However, almost all IDCs with FF having tumor necrosis were categorized as IDCs with tumor necrosis, and recently, Jimenez et al. (19) reported the presence of a large centrally necrotizing area within IDCs to be a very useful prognostic parameter for patients with IDCs. Thus, in this study, we excluded the presence of tumor necrosis within FF from the parameters predicting the outcomes of patients with IDCs with FF.

The maximum, minimum, median, and mean \pm standard deviation of FF dimension in IDCs were 30.0, 1.0, 8.0, and $9.5 \pm 5.8 \mathrm{~mm}$, respectively. Among these characteristics, we established cutoff values for FF dimensions to evaluate significant associations with tumor recurrence, initial distant organ metastasis, and tumor death by univariate and multivariate analyses, because FF dimension in each IDC was evaluated in real numbers. Suitable cutoff values for maximum FF dimension were selected based on the values yielding the lowest $P$ value in univariate analyses for tumor recurrence, initial distant organ metastasis, and tumor death in IDCs with FF. The following cutoff values for maximum FF dimension wêre established: (1) tumor recurrence and initial distant organ metastasis, $\leq 8$ versus $>8$, and (2) tumor death, $\leq 17$ versus $>17 \mathrm{~mm}$. The cut-off values of $\mathrm{FF}$ dimension for tumor recurrence and initial distant organ metastasis, and the median value of FF dimension in all IDCs were the same. The prognostic significance of these FF characteristics was evaluated with the same parameters that were used to examine the relation- ships between the presence of FF and tumor recurrence, initial distant organ metastasis, and tumor death by multivariate analysis in IDCs with FF. The FF characteristics significantly increasing hazard rates (HRs) of tumor recurrence, initial distant organ metastasis, and tumor death on the multivariate analysis were used in a combination assessment of the presence of FF for tumor progression in IDC groups, in which only the presence of FF had no prognostic significance on the multivariate analysis.

All analyses were performed with Statistica/Windows software (StatSoft, Tulsa, OK).

\section{RESULTS}

\section{Clinicopathological Parameters Significantly Associated with the Presence of FF}

The parameters significantly associated with the presence of $\mathrm{FF}$ in the univariate analyses were an invasive tumor size of $>20 \mathrm{~mm}(P<.001)$, histologic Grade $3(P=.018)$, the presence of lymphatic invasion $(P=.002)$, the presence of vascular invasion $(P<$ .001), the presence of adipose tissue invasion $(P<$ $.001)$, the presence of skin invasion $(P=.016)$, and the presence of nodal metastasis $(P=.001)$. Among these parameters, those that still significantly increased the relative risks of the presence of FF were the presence of adipose tissue invasion, invasive tumor size of $>20$ $\mathrm{mm}$, the presence of vascular invasion, and the presence of nodal metastasis (Table 1).

\section{Prognostic Significance of the Presence of FF in All Cases}

Multivariate analyses clearly demonstrated that the presence of FF significantly increased the HRs of tumor recurrence, initial distant organ metasta-

TABLE 1. Multivariate Analyses for Clinicopathological Parameters Significantly Associated with the Presence of FF in IDCs $(n=439)$

\begin{tabular}{|c|c|c|c|c|}
\hline \multirow{2}{*}{ Parameters } & \multirow{2}{*}{ Cases } & \multirow{2}{*}{$\begin{array}{c}\mathrm{FF}+ \\
(\%)\end{array}$} & \multicolumn{2}{|c|}{ Multivariate } \\
\hline & & & RR/95\% CI & $P$ Value \\
\hline \multicolumn{5}{|c|}{ Adipose tissue invasion $(n)$} \\
\hline Absent & 54 & $13(24)$ & Referent & \\
\hline Present & 385 & $222(58)$ & $3.5 / 1.8-6.8$ & $<0.001$ \\
\hline \multicolumn{5}{|c|}{ Invasive tumor size $(n)$} \\
\hline$\leq 20 \mathrm{~mm}$ & 184 & $74(40)$ & Referent & \\
\hline$>20 \mathrm{~mm}$ & 255 & $161(63)$ & $2.1 / 1.4-3.1$ & $<0.001$ \\
\hline \multicolumn{5}{|c|}{ Vascular invasion $(n)$} \\
\hline Absent & 273 & $127(47)$ & Referent & \\
\hline Present & 166 & $108(65)$ & $1.7 / 1.1-2.5$ & 0.018 \\
\hline \multicolumn{5}{|c|}{ Nodal status $(n)$} \\
\hline Negative & 207 & $94(45)$ & Referent & \\
\hline Positive & 232 & $141(61)$ & $1.5 / 1.0-2.3$ & 0.048 \\
\hline
\end{tabular}

Multivariate analyses for the presence of FF were performed using a logistic regression model adjusted for parameters significantly associated with the presence of FF on univariate analysis. The step-down method was applied until all remaining parameters were significant at a $P$ level below 0.05 . The significant parameters were then examined by multivariate analysis.

FF, Fibrotic focus; IDC, invasive ductal carcinoma; Univariate, univariate analysis; Multivariate, multivariate analysis; +, Present; RR, Relative risk; CI, Confidence interval. 
sis, and tumor death (Table 1, Fig. 5). The presence of nodal metastasis and negative ER or PR status also significantly increased the HRs of tumor recurrence, initial distant organ metastasis, and tumor death on multivariate analysis. Although age of $\leq 39$ years significantly increased the HRs of tumor recurrence and initial distant organ metastasis, it had no significant effect on tumor death in multivariate analyses. The presence of lymphatic invasion, invasive tumor size of $>20 \mathrm{~mm}$, and histologic Grade 3 significantly increased the HRs of tumor recurrence, and the presence of tumor necrosis also increased the HRs of initial distant organ metastasis.

A

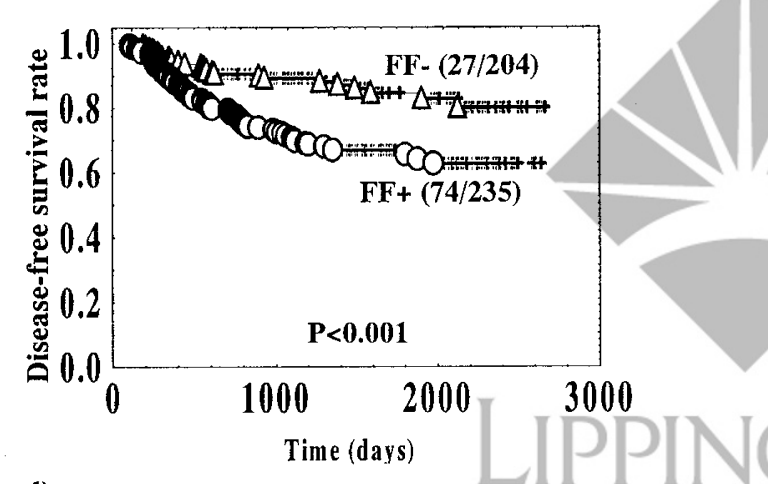

B
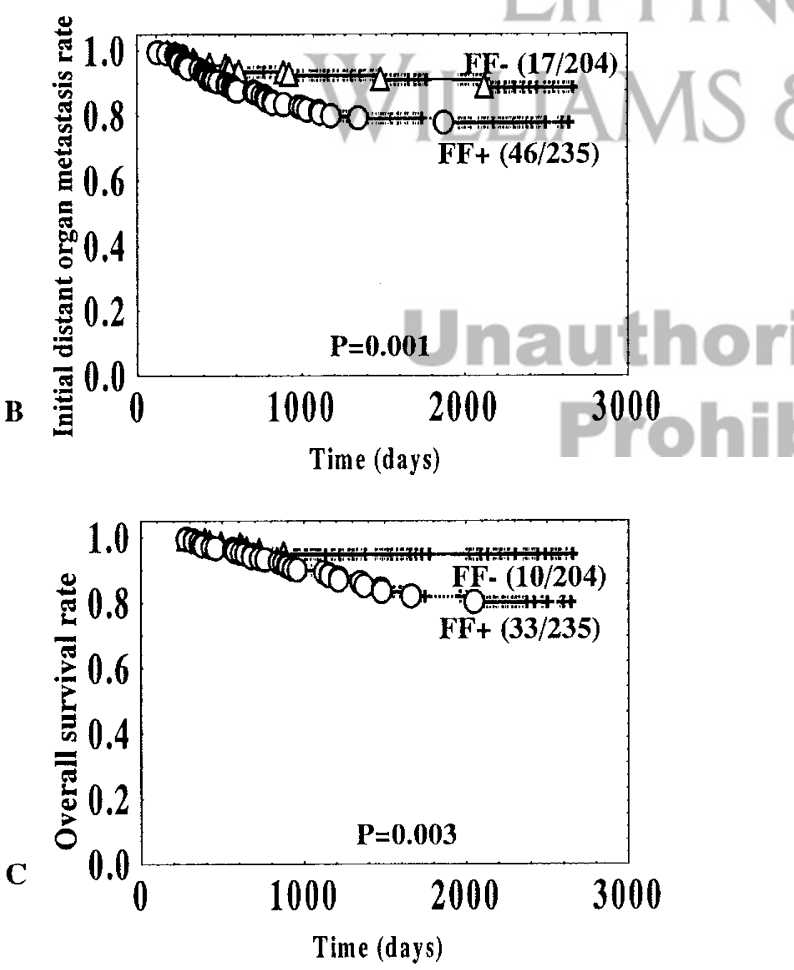

FIGURE 5. A-C, Patients with invasive ductal carcinoma with fibrotic focus (FF) have significantly shorter disease-free survival, initial distant organ metastasis-free survival, and overall survival periods than do those without FF. $(P<.001, P=.001$, and $P=.003$, respectively). - , absent; + , present.
Prognostic Significance of the Presence of FF in Lymph Node-Negative and -Positive Cases

In 207 lymph node-negative cases, the presence of FF was the only parameter that significantly increased the HRs of tumor recurrence and of initial distant organ metastasis on multivariate analysis (Table 1, Fig 6A-B). Age of $\leq 39$ years and positive adjuvant therapy significantly increased the HRs of initial distant organ metastasis and tumor recurrence on multivariate analysis, respectively. Other parameters, such as ER or PR status or histologic grade, had no significant effect on tumor progression in lymph node-negative cases on multivariate analysis (data not shown). As for tumor death, the presence of FF ( $P=.008$, Fig. $6 \mathrm{C})$, and ER and PR both negative $(P<.001)$ were parameters significantly associated with short overall survival periods on univariate analyses.

In 232 lymph node-positive cases, the presence of FF significantly increased the HRs of tumor recurrence (HR, 1.7; 95\% CI, 1.1-2.7; $P=.035$ ) but did not increase the HRs of initial distant organ metastasis and tumor death on multivariate analysis (data not shown). However, negative ER or PR status significantly increased the HRs of tumor recurrence (HR, 0.4; 95\% CI, 0.2-0.6; $P<.001$ ), initial distant organ metastasis (HR, 0.3 ; $95 \% \mathrm{CI}, 0.2-0.6$; $P$ $<.001$ ), and tumor death (HR, 0.2; 95\% CI, 0.1-0.4; $P<.001$ ) on multivariate analysis. The presence of tumor necrosis significantly increased the HRs of tumor recurrence (HR, 2.4; 95\% CI, 1.4-3.9; $P=$ .001 ) and initial distant organ metastasis (HR, 2.5; $95 \% \mathrm{CI}, 1.4-4.6 ; P=.002$ ) on multivariate analysis. Histologic Grade 3 significantly increased the HRs of tumor recurrence (HR, 2.7; 95\% CI, 1.7-4.4; $P<$ .001 ) and tumor death (HR, 4.7; 95\% CI, 2.2-9.1, $P<$ $.001)$ on multivariate analysis. Parameters significantly increasing the HR of tumor recurrence were nodal status of $>3$ (HR, 3.0; 95\% CI, 1.8-4.9; $P<$ .001 ) and the presence of lymphatic invasion (HR, $2.0 ; 95 \% \mathrm{CI}, 1.2-3.4 ; P=.006)$. The parameter that significantly increased the HR of initial distant organ metastasis was age $\leq 39$ years (HR, 0.3 ; 95\% CI, $0.1-0.6 ; P<.001)$ on the multivariate analysis.

Prognostic Significance of the Presence of FF in Cases of Either or Both ER- and PR-Positive and Both ER- and PR-Negative Cases

In cases positive for either or both ER and PR, the presence of FF and invasive size of $>20 \mathrm{~mm}$ significantly increased the HRs of tumor recurrence and initial distant organ metastasis on multivariate analysis (Table 2, Fig. 6D, E). A histologic grade of 3 and the presence of skin invasion significantly increased the HRs of tumor recurrence, and age of $\leq 39$ years significantly increased the HR of initial distant organ metastasis on multivariate analysis 
(Table 2). As for tumor death, the presence of FF ( $P$ $=.042$, Fig. $6 \mathrm{~F})$, invasive size of $>20 \mathrm{~mm}(P=.012)$, histologic Grade $3(P=.010)$, and metastatic lymph nodes $(P=.004)$ were significantly associated with short overall survival periods in univariate analyses.

In cases negative for both ER or PR, although the presence of FF significantly increased the HR of tumor recurrence (HR, 2.0; 95\% CI, 1.1-3.6; $P=$ .027), it did not increase the HRs of initial distant organ metastasis and tumor death on multivariate analysis (data not shown). As for other parameters, the presence of nodal metastasis significantly increased the HRs of tumor recurrence (HR, 4.2; 95\% CI, 2.0-8.8; $P<.001$ ), initial distant organ metastasis (HR, 5.9; 95\% CI, 2.5-14.6; $P<.001$ ), and tumor death (HR, 3.8; 95\% CI, 1.4-9.4; $P<.001$ ) on multivariate analysis. The other parameters that significantly increased the HR of tumor recurrence were the presence of lymphatic invasion (HR, 2.5; 95\% CI, 1.3-4.5; $P=.003$ ), histologic Grade 3 (HR, 2.4; 95\% CI, 1.3-4.3; $P=.003$ ), and tumor necrosis (HR, 1.8; 95\% CI, 1.0-3.3; $P=.038$ ) on multivariate analysis. Among these, the presence of lymphatic invasion and histologic Grade 3 significantly increased the HRs of tumor death on multivariate analysis (HR, 2.4; 95\% CI, 1.2-5.2; $P=.020$, and HR, 4.0; 95\% CI, 2.0-8.3; $P<.001$, respectively). The presence of tumor necrosis and of age $\leq 39$ years significantly increased the HRs of initial distant organ metastasis on multivariate analysis (HR, 2.7;
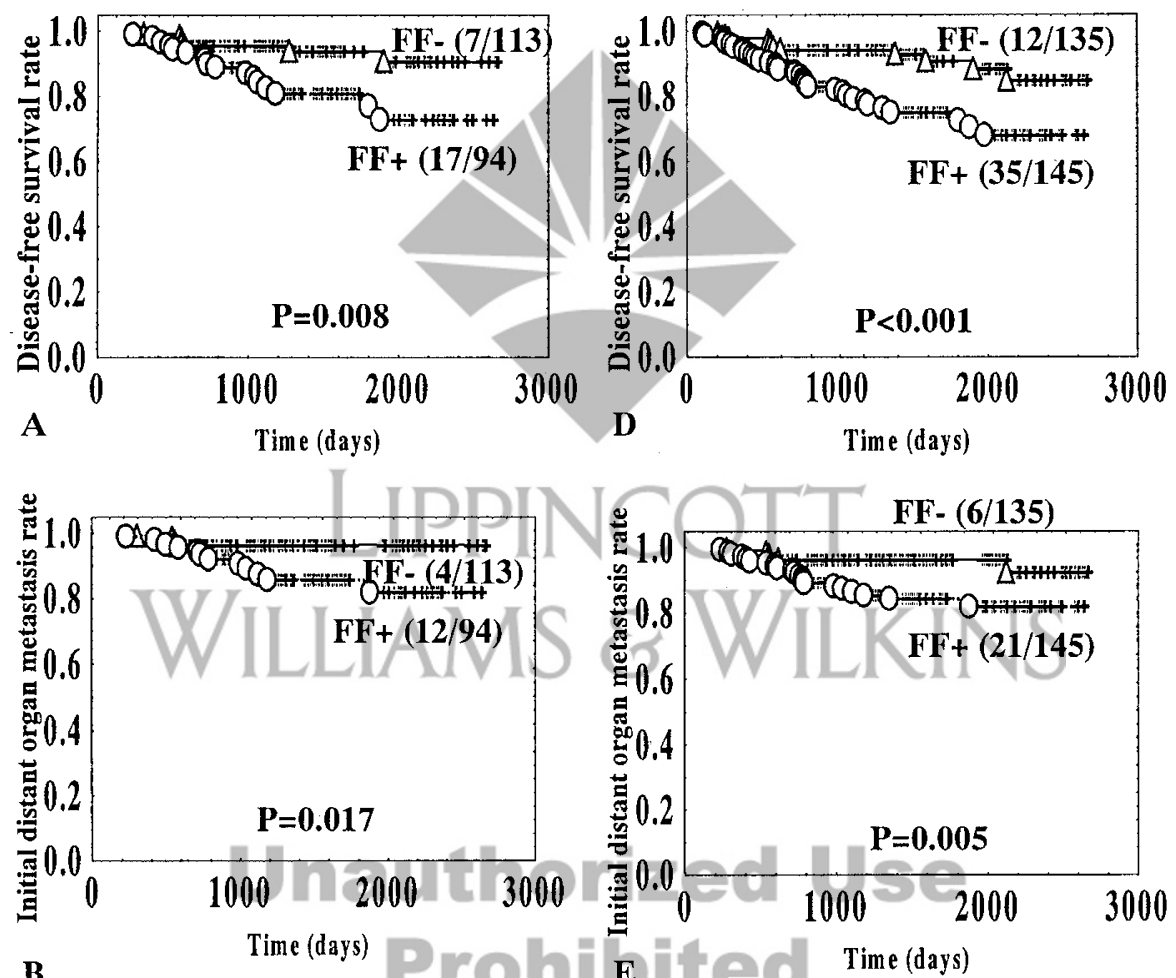

\section{B}

FF- $(0 / 113)$
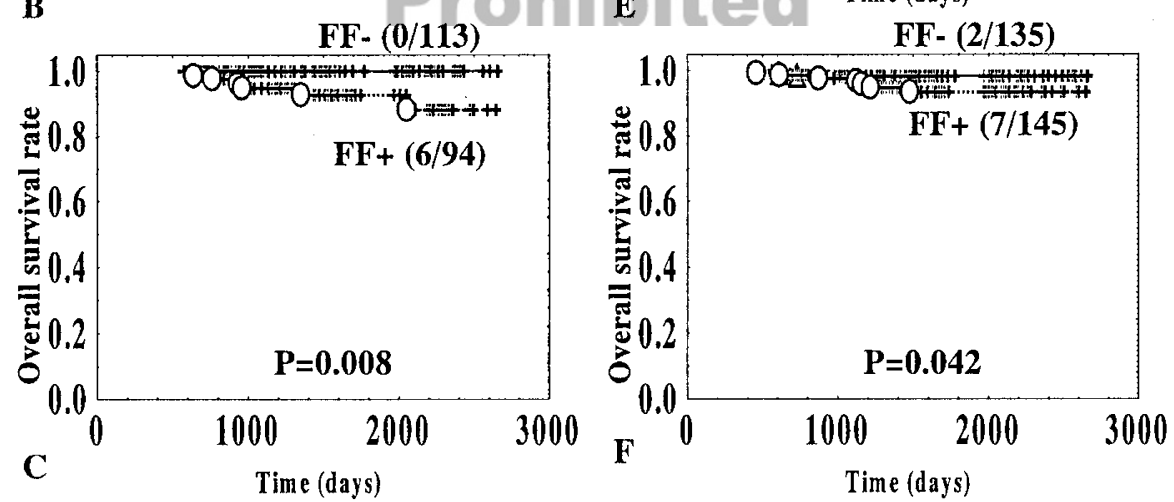

FIGURE 6. Disease-free survivals, initial distant organ metastasis-free survivals, and overall survivals of lymph node-negative invasive ductal carcinoma (IDC) patients (A-C) and of either or both ER- or PR-positive IDC patients (D-F). A-C, as for lymph node-negative IDCs, patients with fibrotic focus (FF) have significantly shorter disease-free, initial distant organ metastasis-free, and overall survival periods than those without FF. ( $P$ $=.008, P=.017$, and $P=.008$, respectively). D-F, among either or both ER- or PR-positive IDCs, patients who have IDCs with FF have significantly shorter disease-free, initial distant organ metastasis-free, and overall survival periods than do those without FF. $(P<.001, P=.005$, and $P=.042$, respectively). -, absent; +, present. 
TABLE 2. Multivariate Analyses for DFS, IDOM, and OS in All IDCs, Lymph Node-Negative IDCs, and ER/PR Either or Both Positive IDCs

\begin{tabular}{|c|c|c|c|c|c|c|c|}
\hline \multirow{2}{*}{ Parameters } & \multirow{2}{*}{ Cases } & \multicolumn{6}{|c|}{$\mathrm{HR} / 95 \% \mathrm{CI}$} \\
\hline & & TRR (\%) & $P$ Value & IDOMR (\%) & $P$ Value & MR (\%) & $P$ Value \\
\hline \multicolumn{8}{|c|}{ All IDCs $(n=439)$} \\
\hline \multicolumn{8}{|c|}{ Fibrotic focus } \\
\hline Absent & 204 & $27(13)$ & Referent & $17(8)$ & Referent & $10(5)$ & Referent \\
\hline Present & 235 & $74(31)$ & $\begin{array}{l}2.0 / 1.3-3.1 \\
0.003\end{array}$ & $\begin{array}{r}46(20) \\
0.021\end{array}$ & $1.9 / 1.1-3.4$ & $33(14)$ & $\begin{array}{l}2.3 / 1.1-4.7 \\
0.019\end{array}$ \\
\hline \multicolumn{8}{|l|}{ Nodal status } \\
\hline Negative & 207 & $24(11)$ & Referent & $16(7)$ & Referent & $6(3)$ & Referent \\
\hline Positive & 232 & 77 (33) & $\begin{array}{l}2.7 / 1.6-4.4 \\
<0.001\end{array}$ & $47(20)$ & $\begin{array}{l}2.9 / 1.6-5.2 \\
<0.001\end{array}$ & $37(16)$ & $\begin{array}{l}5.5 / 2.3-13 \\
<0.001\end{array}$ \\
\hline \multicolumn{8}{|l|}{ ER/PR status } \\
\hline- & 159 & $54(34)$ & Referent & $36(23)$ & Referent & $34(21)$ & Referent \\
\hline+ & 280 & $47(17)$ & $\begin{array}{l}0.5 / 0.3-0.7 \\
<0.001\end{array}$ & $27(10)$ & $\begin{array}{l}0.5 / 0.3-0.9 \\
0.007\end{array}$ & $9(3)$ & $\begin{array}{l}0.1 / 0.1-0.3 \\
<0.001\end{array}$ \\
\hline \multicolumn{8}{|l|}{ Age $(\mathrm{yr})$} \\
\hline$\leq 39$ & 36 & $17(47)$ & Referent & $14(39)$ & Referent & $8(22)$ & Referent \\
\hline$>39$ & 403 & $84(21)$ & $\begin{array}{l}0.6 / 0.3-0.9 \\
0.040\end{array}$ & 49 (12) & $\begin{array}{l}0.3 / 0.2-0.5 \\
<0.001\end{array}$ & $35(9)$ & $--/--$ \\
\hline \multicolumn{8}{|c|}{ Lymphatic invasion } \\
\hline Absent & 254 & $42(16)$ & Referent & $27(11)$ & Referent & $16(6)$ & Referent \\
\hline Present & 185 & $59(32)$ & $\begin{array}{l}1.6 / 1.0-2.5 \\
0.036\end{array}$ & $36(19)$ & $--/--$ & $24(15)$ & $--/--$ \\
\hline \multicolumn{8}{|c|}{ Invasive tumor size (mm) } \\
\hline$\leq 20$ & 184 & $22(12)$ & Referent & $14(8)$ & Referent & $10(5)$ & Referent \\
\hline$>20$ & 255 & $79(31)$ & $\begin{array}{l}1.7 / 1.1-2.8 \\
0.029\end{array}$ & $49(19)$ & $--/--$ & $33(13)$ & $--/--$ \\
\hline \multicolumn{8}{|c|}{ Histologic grade } \\
\hline 1 and 2 & 307 & $50(16)$ & Referent & $33(11)$ & Referent & $15(5)$ & Referent \\
\hline 3 & 132 & $51(39)$ & $\begin{array}{l}2.4 / 1.6-3.6 \\
<0.001\end{array}$ & $30(23)$ & $--/--$ & $28(21)$ & $--/--$ \\
\hline \multicolumn{8}{|c|}{ Tumor necrosis } \\
\hline Absent & 332 & $61(18)$ & Referent & $36(11)$ & Referent & $24(7)$ & Referent \\
\hline Present & 107 & $40(37)$ & $\begin{array}{l}-/- \\
0.001\end{array}$ & $27(25)$ & $2.4 / 1.3-4.0$ & $19(18)$ & $--/--$ \\
\hline \multicolumn{8}{|c|}{ Lymph node-negative IDCs ( $n=207)$} \\
\hline Fibrotic focu & & & & & & & \\
\hline Absent & 113 & $7(6)$ & Referent & $4(4)$ & Referent & & \\
\hline Present & 94 & $17(18)$ & $\begin{array}{l}2.8 / 1.1-6 \\
0.024\end{array}$ & $12(13)$ & $\begin{array}{l}3.6 / 1.2-11.2 \\
0.026\end{array}$ & & \\
\hline Age (yr) & & & & & & & \\
\hline$\leq 39$ & 16 & $5(31)$ & & & Referent & & \\
\hline & & & & & & & \\
\hline Adjuvant the & & & & & & & \\
\hline No & 99 & $5(5)$ & Referent & $4(4)$ & NS & & \\
\hline Yes & 108 & $19(18)$ & $\begin{array}{l}3.2 / 1.2-8.8 \\
0.021\end{array}$ & $12(11)$ & & & \\
\hline ER/PR either o & ositive II & $=280)$ & & & & & \\
\hline Fibrotic focu & & & & & & & \\
\hline Absent & 135 & $12(9)$ & Referent & $6(4)$ & Referent & & \\
\hline Present & 145 & $35(24)$ & $2.5 / 1.3-4.8$ & $21(14)$ & $3.2 / 1.2-8.1$ & & \\
\hline Invasive tum & $\mathrm{mm})$ & & 0.007 & & 0.015 & & \\
\hline$\leq 20$ & 118 & $8(7)$ & Referent & $4(3)$ & Referent & & \\
\hline$>20$ & 162 & $39(24)$ & $2.3 / 1.0-5.1$ & $23(14)$ & $3.5 / 1.2-10.2$ & & \\
\hline & & & 0.044 & & 0.024 & & \\
\hline Histologic gr & & & & & & & \\
\hline 1 and 2 & 222 & $28(13)$ & Referent & $17(8)$ & Referent & & \\
\hline 3 & 58 & $19(33)$ & $\begin{array}{l}2.3 / 1.2-4.2 \\
0.007\end{array}$ & $10(17)$ & $--/--$ & & \\
\hline Skin invasion & & & & & & & \\
\hline Absent & 239 & $33(14)$ & Referent & $20(8)$ & NS & & \\
\hline Present & 41 & $14(34)$ & $\begin{array}{l}2.0 / 1.0-3.7 \\
0.040\end{array}$ & $7(17)$ & & & \\
\hline Age (yr) & & & & & & & \\
\hline$\leq 39$ & 20 & $6(30)$ & NS & $5(25)$ & Referent & & \\
\hline$>39$ & 260 & $41(16)$ & & $22(9)$ & $0.3 / 0.1-0.8$ & & \\
\hline & & & & & 0.019 & & \\
\hline
\end{tabular}

DFS, Disease-free survival; IDOM, initial distant organ metastasis; OS, Overall survival; HR, Hazard rate; CI, Confidence interval; TRR, Tumor recurrence rate; IDOMR, DOM rate; MR, Mortality rate; FF, Fibrotic focus; ER, Estrogen receptor; PR, Progesterone receptor; +, Either or both positive; -, Both negative; NS, Not significant on univariate analysis; --/--, Not significant on multivariate analysis. The multivariate analyses for TRR, IDOMR, and MR were performed using the Cox proportional hazard regression model adjusted for parameters significantly associated with TRR, IDOMR, and MR in the univariate analyses. The step-down method, until all the remaining parameters were significant at a $P$ level of $<0.05$, was used for the multivariate analysis. 
95\% CI, $1.4-5.3 ; P=.003$ and HR, $0.3 / 95 \%$ CI, $0.1-0.6 ; P<.001$, respectively).

Prognostic Significance of the Presence of FF in Lymph Node-Negative Cases and Cases Either or Both ER or PR Positive

In lymph node-negative cases and those positive for either or both ER or PR, despite IDCs with FF showing significantly shorter disease-free survival and initial distant organ metastasis-free survival periods than those without FF $(P=.037$ and $P=$ .029 , respectively), the presence of FF did not increase the HRs of tumor recurrence and initial organ metastasis on multivariate analysis (data not shown). Multivariate analyses showed adjuvant therapy to significantly increase the HR of tumor recurrence (HR, 5.8; 95\% CI, 1.3-27.9; $P=.023$ ), and the presence of tumor necrosis also increased the HRs of initial distant organ metastasis (HR, 5.3; 95\% CI, 1.4-21.6; $P=.016$ ).

\section{Prognostic Significance of FF Characteristics}

In IDCs with FF, on multivariate analysis, FF dimension of $>8 \mathrm{~mm}$ and of $>17 \mathrm{~mm}$ significantly increased the HRs of initial distant organ metastasis and tumor death, respectively (Table 3). The fibrosis grade of FF had no significant association with tumor progression of IDCs with FF (data not shown). As for other parameters, the presence of nodal metastasis significantly increased the HRs of tumor recurrence, initial distant organ metastasis, and tumor death on multivariate analysis (Table 3 ). Age of $\leq 39$ years and invasive tumor size of $>20$ mm significantly increased the HRs of tumor recurrence and initial distant organ metastasis on multivariate analysis. Histologic Grade 3 and both ER and PR negative significantly increased the HRs of tumor recurrence and of death on multivariate analysis.

\section{Combined Assessment of the Presence of FF and FF Dimension}

In lymph node-positive cases, IDCs with FF of $>8$ $\mathrm{mm}$ in dimension were associated with significant increases in the HRs of tumor recurrence and initial distant organ metastasis on multivariate analysis (Table 4, Fig. 7A, B). In addition, IDCs with FF of $>17 \mathrm{~mm}$ in dimension showed a significant increase in the HR of tumor death on multivariate analysis (Fig. 7C). As for other parameters, both ER

TABLE 3. Multivariate Analyses for DPS, IDOM, and OS in IDCs with FF

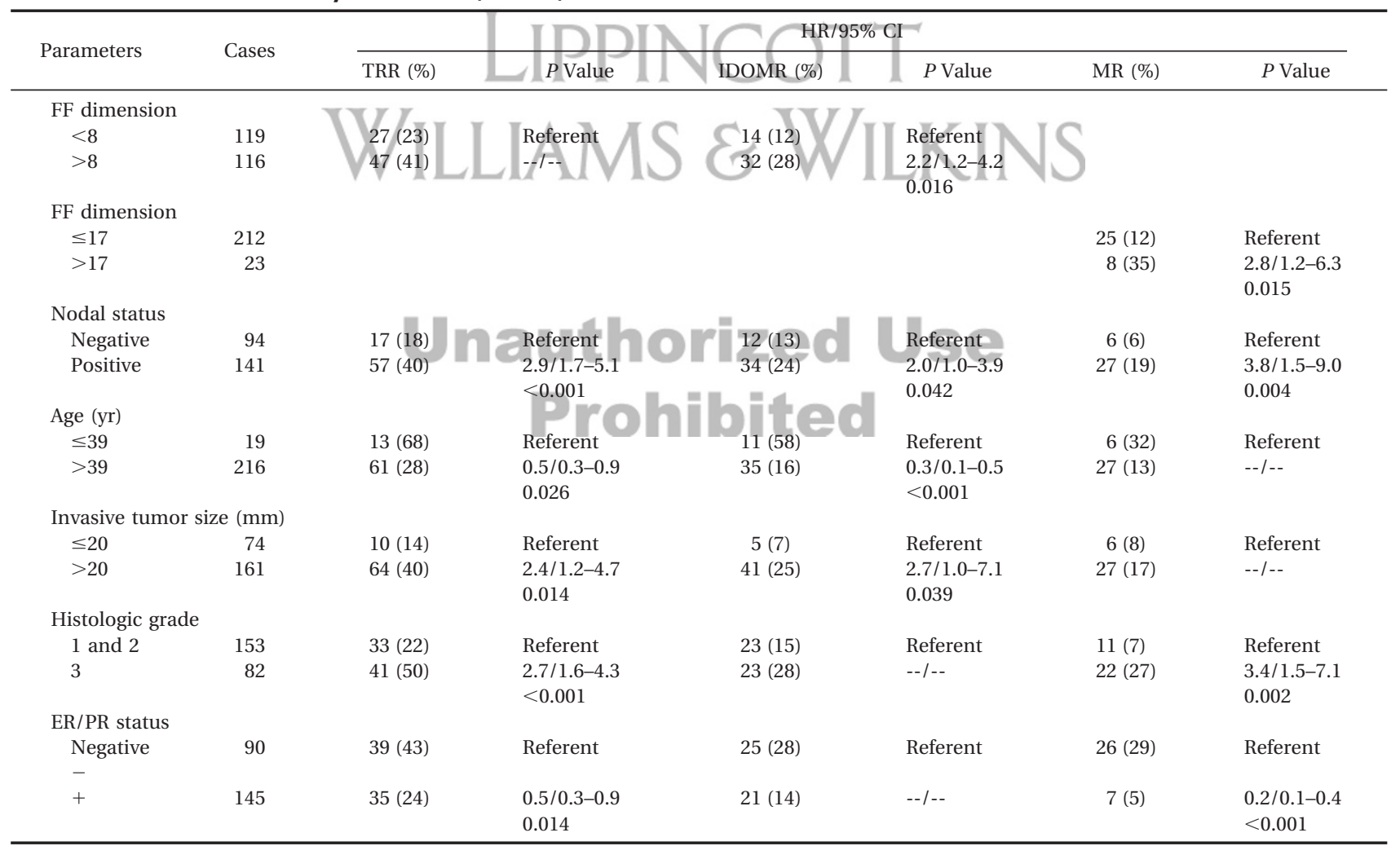

DFS, Disease-free survival; IDOM, Initial distant organ metastasis; OS, Overall survival; HR, Hazard rate; CI, Confidence interval; TRR, Tumor recurrence rate; IDOMR, IDOM rate; MR, Mortality rate; FF. Fibrotic focus; ER, Estrogen receptor; PR, Progesterone receptor; +, Either or both positive; -, Both negative; NS, Not significant on univariate analysis; --/--, Not significant on multivariate analysis. The multivariate analyses for TRR, IDOMR, and MR were performed using the Cox proportional hazard regression model adjusted for parameters significantly associated with TRR, IDOMR, and MR in the univariate analyses. The step-down method, until all the remaining parameters were significant at a p-level below 0.05 , was used for the multivariate analysis. 
and PR negative significantly increased the HRs of tumor recurrence, initial distant organ metastasis, and tumor death on multivariate analysis. The presence of tumor necrosis significantly increased the HRs of tumor recurrence and initial distant organ metastasis on multivariate analysis. Histologic Grade 3 significantly increased the HRs of tumor recurrence and death on multivariate analysis. Nodal metastases of more than three significantly increased the HR of tumor recurrence on multivariate analysis.

In cases negative for both ER or PR, IDCs with FF of $>8 \mathrm{~mm}$ and those with FF of $>17 \mathrm{~mm}$ were significantly associated with significantly increased HRs of tumor recurrence and tumor death on multivariate analysis (Table 4, Fig. 7D, E). Positive nodal metastasis significantly increased the HRs of tumor recurrence, initial distant organ metastasis, and tumor death on multivariate analysis, and histologic Grade 3 significantly increased the HRs of tumor recurrence and death on multivariate analysis. The parameters significantly increasing the HRs of tumor recurrence were the presence of lymphatic invasion and the presence of vascular invasion, and those that significantly increased the HRs of initial distant organ metastasis were age $\leq 39$ years and the presence of tumor necrosis.

In lymph node-negative and either or both ER- or PR-positive cases, FF dimension of $>8 \mathrm{~mm}$ significantly increased the HRs of tumor recurrence and initial distant organ metastasis on multivariate analysis (Table 4, Fig 7F, G). Although the presence of tumor necrosis significantly increased the HR of initial distant organ metastasis, it did not increase the HR of tumor recurrence on multivariate analysis. Adjuvant therapy produced no significant increase in the HR of tumor recurrence on multivariate analysis (data not shown).

\section{DISCUSSION}

The parameters significantly associated with the presence of FF were the presence of adipose tissue invasion, invasive tumor size of $>20 \mathrm{~mm}$, the presence of vascular invasion, and the presence of nodal metastasis. The significant associations of the presence of FF with both adipose tissue invasion, and invasive tumor size of $>20 \mathrm{~mm}$ probably suggest that IDCs with FF have a greater aggressive invasive potential than do IDCs without FF. The significant associations of the presence of FF with both vascular invasion and nodal metastasis most likely reflect IDCs with FF having a higher metastatic potential than IDCs without FF. In addition, we have already clarified that the proliferative activity of the tumor cells and tumor angiogenesis are significantly higher in IDCs with than in those with- out FF $(8,9)$. Therefore, IDCs with FF are likely to be more invasive and more metastatic than IDCs without FF.

The current prospective study clearly demonstrated FF dimension to be a very useful prognostic parameter for patients with IDCs with FF. Although we have previously reported FF dimension not to be a significant prognostic parameter for patients with IDCs with FF $(6,7)$, the cutoff value of FF dimension for predicting the outcomes of patients with IDCs with FF was not optimal in previous studies. In addition, there were fewer cases in the earlier than in the present study. Thus, a significant association between FF dimension and the outcomes of patients with IDCs having FF could not be identified in previous studies. The observations made in the current prospective study allowed us to set appropriate cutoff values of FF dimensions for classifying patients with IDCs with FF into low-risk and high-risk groups: (1) tumor recurrence and initial distant organ metastasis, $8 \mathrm{~mm}$ and (2) tumor death, $17 \mathrm{~mm}$.

The current prospective study showed the presence of FF to be a very useful histologic prognostic parameter for patients with node-negative IDCs, as well as for patients with either or both ER- or PRpositive IDCs and all IDC patients. However, the presence of FF had no prognostic significance in patients with node-positive IDCs, those with both ER- and PR-negative IDCs, or those with nodenegative and either or both ER- or PR-positive IDCs. IDCs can probably be categorized into the following groups: (1) very low-risk group, (2) low-risk group, and (3) high-risk group. Furthermore, nodenegative IDCs, and those that are either or both ERor PR-positive, belong to the low-risk group. Thus, the presence of FF is probably an independent prognostic parameter for patients with low-risk IDCs. However, the combined assessment of the presence of FF and FF dimension enabled us to accurately predict outcomes of patients with nodepositive IDCs, those with both ER- and PR-negative IDCs, and those with either or both ER- or PRpositive and node-negative IDCs. Thus, we conclude that this combined assessment can accurately predict outcomes of patients with IDCs belonging to all categories.

We have already demonstrated highly proliferative fibroblasts forming FF to play an important role in tumor progression of IDCs with FF (9). Thus, it seems reasonable that FF dimension is a valuable parameter for predicting outcomes of patients with IDCs with FF. The cutoff value for FF dimension as a predictor of tumor death of patients with IDCs with FF was larger than the cutoff values for FF dimension as a predictor of tumor recurrence and initial distant organ metastasis in this study. This strongly suggests that the lethality of FF originates from the abundant stromal 
TABLE 4. Multivariate Analyses for DFS, IDOM, and OS in Lymph Node Positive IDCs, ER/PR Both Negative IDCs, and Lymph Node Negative and ER/PR Either or Both Positive IDCs

\begin{tabular}{|c|c|c|c|c|c|c|c|}
\hline \multirow{2}{*}{ Parameters } & \multirow{2}{*}{ Cases } & \multicolumn{6}{|c|}{$\mathrm{HR} / 95 \% \mathrm{CI}$} \\
\hline & & TRR (\%) & $\mathrm{P}$ Value & IDOMR (\%) & $P$ Value & MR (\%) & P Value \\
\hline \multicolumn{8}{|c|}{ Lymph node positive IDCs $(n=232)$} \\
\hline \multicolumn{8}{|c|}{ Fibrotic focus, dimension (mm) } \\
\hline$-/<8$ & 164 & $44(27)$ & Referent & $25(15)$ & Referent & & \\
\hline \multirow[t]{2}{*}{$>8$} & 68 & $33(49)$ & $1.6 / 1.0-2.5$ & $22(32)$ & $1.9 / 1.1-3.4$ & & \\
\hline & & & 0.047 & & 0.034 & & \\
\hline \multicolumn{8}{|c|}{ Fibrotic focus, dimension (mm) } \\
\hline$-/ \leq 17$ & 213 & & & & & $29(14)$ & Referent \\
\hline$>17$ & 19 & & & & & $8(42)$ & $\begin{array}{l}3.1 / 1.4-6.7 \\
0.006\end{array}$ \\
\hline \multicolumn{8}{|c|}{ ER/PR status } \\
\hline- & 87 & $44(51)$ & Referent & $30(34)$ & Referent & $28(32)$ & Referent \\
\hline+ & 145 & $33(23)$ & $0.4 / 0.2-0.6$ & $17(12)$ & $0.3 / 0.2-0.6$ & $9(6)$ & $0.2 / 0.1-0.4$ \\
\hline \multicolumn{8}{|c|}{ Tumor necrosis } \\
\hline Absent & 177 & $47(27)$ & Referent & $27(15)$ & Referent & $20(11)$ & Referent \\
\hline Present & 55 & $30(55)$ & $\begin{array}{l}2.0 / 1.2-3.4 \\
0.007\end{array}$ & $20(36)$ & $\begin{array}{l}2.2 / 1.2-4.0 \\
0.010\end{array}$ & $17(31)$ & $--/--$ \\
\hline \multicolumn{8}{|c|}{ Histologic grade } \\
\hline 1 and 2 & 159 & $37(23)$ & Referent & $25(16)$ & Referent & $12(8)$ & Referent \\
\hline 3 & 73 & $40(55)$ & $\begin{array}{l}2.6 / 1.6-4.2 \\
<0.001\end{array}$ & $22(30)$ & $--/--$ & $25(34)$ & $\begin{array}{l}4.3 / 2.2-8.8 \\
<0.001\end{array}$ \\
\hline \multicolumn{8}{|c|}{ Nodal status } \\
\hline$\leq 3$ & 131 & $30(23)$ & Referent & $24(18)$ & NS & $17(13)$ & NS \\
\hline$>3$ & 101 & $47(47)$ & $3.2 / 1.9-5.3$ & $23(23)$ & & $20(19)$ & \\
\hline ER/PR both & e IDCs & & & & & & \\
\hline Fibrotic fo & mensior & & & & & & \\
\hline$-/ \leq 8$ & 106 & $27(25)$ & Referent & $16(15)$ & Referent & & \\
\hline$>8$ & 53 & $27(51)$ & $2.0 / 1.1-3.6$ & $20(38)$ & $--/--$ & & \\
\hline Fibrotic fo & mensior & & & & & & \\
\hline$-/ \leq 17$ & 148 & & & & & $27(18)$ & Referent \\
\hline$>17$ & 11 & & & & & $7(64)$ & $\begin{array}{l}3.3 / 1.4-7.7 \\
0.008\end{array}$ \\
\hline Nodal stat & & & & & & & \\
\hline Absent & 72 & $10(14)$ & Referent & $6(8)$ & Referent & $6(8)$ & Referent \\
\hline Present & 87 & $44(51)$ & $3.7 / 1.8-7.6$ & $30(35)$ & $5.9 / 2.4-14.0$ & $28(32)$ & $4.7 / 1.9-12$ \\
\hline & & & $<0.001$ & & $<0.001$ & & $<0.001$ \\
\hline Histologic & & & & & & & \\
\hline 1 and 2 & 85 & $22(26)$ & Referent & 160 & NS & $11(13)$ & Referent \\
\hline 3 & 74 & $32(43)$ & $\begin{array}{l}2.7 / 1.5-4.8 \\
<0.001\end{array}$ & & & $23(31)$ & $\begin{array}{l}3.2 / 1.5-6.7 \\
<0.001\end{array}$ \\
\hline Lymphatic & & & & & & & \\
\hline Absent & 96 & $21(22)$ & Referent & 14 (15) & Referent & $12(13)$ & Referent \\
\hline Present & 63 & $33(52)$ & $2.4 / 1.4-4.4$ & 22 (35) & $--/--$ & $22(35)$ & $--/--$ \\
\hline & & & 0.003 & & & & \\
\hline Age (yr.) & & & & & & & \\
\hline$\leq 39$ & 16 & $11(69)$ & Referent & $9(56)$ & Referent & $7(44)$ & Referent \\
\hline$>39$ & 143 & $43(30)$ & $-1--1$ & 27 (19) & $0.3 / 0.1-0.6$ & 27 (19) & $--/--$ \\
\hline Tumor nec & & & & & & & \\
\hline Absent & 95 & 27 (28) & Referent & $16(17)$ & Referent & 17 (18) & NS \\
\hline Present & 64 & $27(42)$ & $-1--$ & $20(31)$ & $2.7 / 1.5-4.9$ & $17(27)$ & \\
\hline & & & & & 0.003 & & \\
\hline Vascular in & & & & & & & \\
\hline Absent & 100 & $29(29)$ & Referent & 19 (19) & NS & 19 (19) & NS \\
\hline Present & 59 & $25(42)$ & $1.8 / 1.0-3.1$ & 17 (29) & & $15(25)$ & \\
\hline & & & 0.046 & & & & \\
\hline Lymph node & ve and & either or b & ositive cases & & & & \\
\hline Fibrotic fo & mensior & & & & & & \\
\hline$-1 \leq 8$ & 109 & $6(8)$ & Referent & $4(4)$ & Referent & & \\
\hline$>8$ & 26 & $8(31)$ & $4.7 / 1.7-10.0$ & $6(23)$ & $4.8 / 1.3-17.4$ & & \\
\hline & & & 0.005 & & 0.018 & & \\
\hline Tumor nec & & & & & & & \\
\hline Absent & 116 & $9(8)$ & Referent & $5(4)$ & Referent & & \\
\hline Present & 19 & $5(26)$ & $--/--$ & $5(26)$ & $5.6 / 1.5-19.6$ & & \\
\hline & & & & & 0.008 & & \\
\hline
\end{tabular}

DFS, Disease-free survival; IDOM, Distant organ metastasis; OS, Overall survival; HR, Hazard rate; CI, Confidence interval; TRR, Tumor recurrence rate; IDOMR, IDOM rate; MR, Mortality rate; FF, Fibrotic focus; $-\mid \leq 8$, Cases without FF and cases with FF $\leq 8 \mathrm{~mm}$ in dimension; $>8$, Cases with FF $>8$ $\mathrm{mm}$ in dimension; $-/ \leq 17$, Cases without FF and cases with $\mathrm{FF} \leq 17 \mathrm{~mm}$ in dimension; $>17$, Cases with FF $>17 \mathrm{~mm}$ in dimension; ER, Estrogen receptor; PR, Progesterone receptor; +, Either or both positive; -, Both negative; NS, Not significant on univariate analysis; --/--, Not significant on multivariate analysis. The multivariate analyses for TRR, IDOMR, and MR were performed using the Cox proportional hazard regression model adjusted for parameters significantly associated with TRR, IDOMR, and MR in the univariate analyses. The step-down method, until all the remaining parameters were significant at a p-level below 0.05 , was used for the multivariate analysis. 

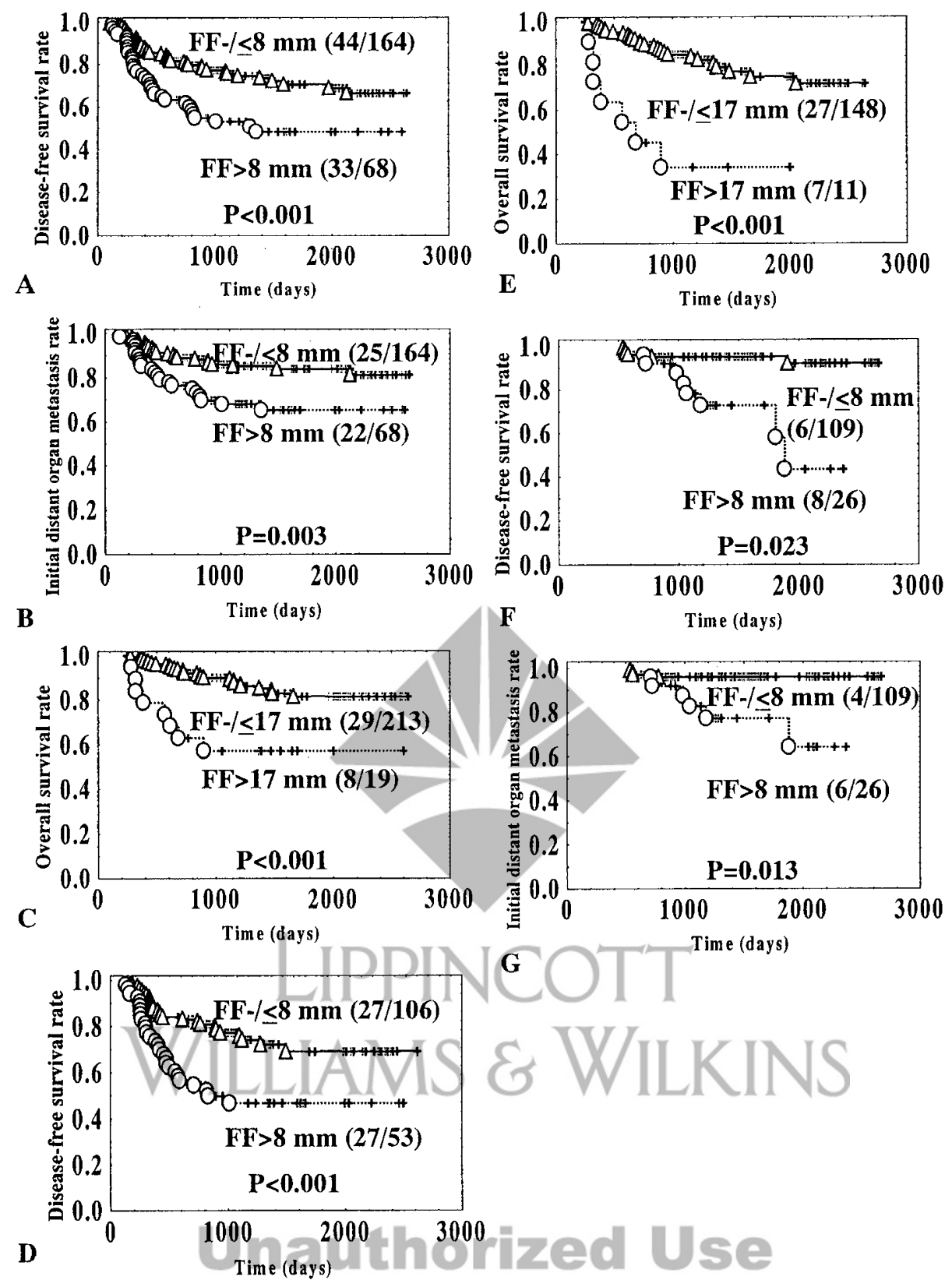

FIGURE 7. Disease-free, initial distant organ metastasis-free, and overall survivals of lymph node-positive invasive ductal carcinomas (IDC) patients (A-C), both estrogen receptor (ER)- and progesterone receptor (PR)-negative IDC patients (D, E), and lymph node-negative and either or both ER- or PR-positive IDC patients (F, G). A-C, as for lymph node-positive IDCs, those with fibrotic focus (FF) of $>8$ mm show significantly shorter disease-free and initial distant organ metastasis-free survival periods than do those without FF and those with FF of $\leq 8$ mm $(P<.001$ and $P$ $=.003$ ). Patients with IDCs with FF of $>17 \mathrm{~mm}$ also show a significantly shorter overall survival period than those without FF and those with FF of $\leq 17 \mathrm{~mm}(P<.001)$. D, E, as for ER or PR both negative IDCs, those with FF of $>8 \mathrm{~mm}$ and those with FF of $>17$ mm show significantly shorter disease-free and overall survival periods than do those without FF or with FF of $\leq 8 \mathrm{~mm}$ and those without FF or with FF of $\leq 17 \mathrm{~mm}(P<.001$ and $P<.001)$. F, G, as for lymph node-negative and either or both ER- or PR-positive IDCs, those with FF of $>8$ mm show significantly shorter diseasefree and initial distant organ metastasis-free survival periods than do those without $\mathrm{FF}$ and those with $\mathrm{FF}$ of $\leq 8 \mathrm{~mm}(P=.023$, and $P=.013)$. FF $-/$ $\leq 8$, IDCs without FF and those with FF of $\leq 8 \mathrm{~mm}$; FF $>8 \mathrm{~mm}$, IDCs with FF of $>8 \mathrm{~mm}$; FF $-/ \leq 17 \mathrm{~mm}$, IDCs without FF and those with FF of $\leq 17$ $\mathrm{mm}$; FF $>17 \mathrm{~mm}$, IDCs with FF of $>17 \mathrm{~mm}$.

components, such as fibroblasts and endothelial cells, that comprise FF $(9,20,21)$.

In conclusion, the current prospective study has clearly shown the presence of FF as well as FF dimension to be a very useful histologic prognostic parameter for patients with IDCs. This strongly suggests that the stromal components making up FF play a very important role in tumor progression of IDCs $(9,20-22)$. Therefore, our next study will focus on why the presence of FF is a useful prognostic parameter for patients with IDCs, especially from the viewpoint of the characteristics of the stromal components comprising FF. 


\section{REFERENCES}

1. Linell F, Ljungberg O, Andersson I. Breast carcinoma: aspects of early stages, progression, and related problems. Acta Pathol Microbiol Scand Suppl 1980;272:1-231.

2. Flsher ER, Palekar AS, Sass R, Fisher B. Scar cancers: pathologic findings from the National Surgical Adjuvant Breast Project (protocol No. 4)-IX. Breast Cancer Res Treat 1983;3: 39-59.

3. Partanen S, Hyvarinen H. Scar and non-scar ductal cancer of the female breast. Observations on patient age, tumour size, and hormone receptors. Virchows Arch 1987;412:145-9.

4. Toikkanen S, Joensuu H. Long-term prognosis of scar and non-scar cancers of the breast. APMIS 1990;98:1033-8.

5. Hasebe T, Tsuda H, Hirohashi S, Shimosato Y, Iwai M, Imoto S, Mukai K. Fibrotic focus in invasive ductal carcinoma: an indicator of high tumor aggressiveness. Jpn J Cancer Res 1996;87:385-94.

6. Hasebe T, Tsuda H, Tsubono Y, Imoto S, Mukai K. Fibrotic focus in invasive ductal carcinoma: a histopathological prognostic parameter for tumor recurrence and tumor death within three years after the initial operation. Jpn J Cancer Res 1997;88:590-9.

7. Hasebe T, Tsuda H, Hirohashi S, Shimosato Y, Tsubono Y, Yamamoto $\mathrm{H}$, et al. Fibrotic focus in infiltrating ductal carcinoma of the breast: a significant histopathological prognostic parameter for predicting the long-term survival of the patients. Breast Cancer Res Treat 1998;49:195-208.

8. Jitsuiki Y, Hasebe T, Tsuda H, Imoto S, Tsubono Y, Sasaki S, et al. Optimizing microvessel counts according to tumor zone in invasive ductal carcinoma of the breast. Mod Pathol 1999;12:492-8.

9. Hasebe T, Sasaki S, Imoto S, Ochiai A. Highly proliferative fibroblasts forming fibrotic focus govern metastasis of invasive ductal carcinoma of the breast. Mod Pathol 2001;14:32537.

10. Colpaert C, Vermeulen P, Jeuris W, van Beest P, Goovaerts G, Weyler J, et al. Early distant relapse in 'node-negative' breast cancer patients is not predicted/by occult axillary lymph node metastases, but by the features of the primary tumour J Pathol 2001;193:442-9.

11. Tsuda H, Takarabe T, Hasegawa T, Murata T, Hirohashi S. Myoepithelial differentiation in high-grade invasive ductal carcinoma with large central acellular zone. Hum Pathol 1999;30:1134-9.

12. Tsuda H, Takarabe T, Hasegawa F, Fukutomi T, Hirohashi S. Large, central acellular zones indicating myoepithelial tumor differentiation in high-grade invasive ductal carcinoma as markers of predisposition to lung and brain metastasis. Am J Surg Pathol 2000;24:197-202.

13. Sobin LH, Witteklnd CH, editors. TNM classification of malignant tumors. 5th ed. New York: Wiley-Liss; 1997.

14. World Health Organization. Histological typing of breast tumors. International histological classification of tumors. 2nd ed. Geneva, Switzerland: World Health Organization; 1981.

15. Elston CW, Ellis IO. Pathological prognostic factors in breast cancer. I. The value of histological grade in breast cancer: experience from a large study with long-term follow-up. Histopathology 1991;19:403-10.

16. Truett J, Cornfield J, Kannel W. A multivariate analysis of the risk of coronary heart disease in Framingham. J Chron Dis 1967;20:511-24.

17. Cox DR. Regression models and life-tables. J R Stat Soc 1972;34:187-220.

18. Kaplan EL, Meier P. Nonparametric estimation from incomplete observations. J Am Stat Assoc 1958;53:457-81.

19. Jimenez RE, Wallis T, Visscher DW. Centrally necrotizing carcinomas of the breast. A distinct histologic subtype with aggressive clinical behavior. Am J Surg Pathol 2001; 25:331-7.

20. Hasebe T, Sasaki S, Imoto S, Ochiai A. Proliferative activity of intratumoral fibroblasts is closely correlated with lymph node and distant organ metastasis of invasive ductal carcinoma of the breast. Am J Pathol 2000;156:1701-10.

21. Hasebe T, Sasaki S, Sugitoh M, Ono M, Saito N, Ochiai A. Highly proliferative intratumoral fibroblasts and a high proliferative microvessel index are significant predictors of tumor metastasis in T3 ulcerative-type colorectal cancer. Hum Pathol 2001;32:401-9.

22. Hasebe T, Imoto S, Ogura $T$, Mukai K. Significance of basic fibroblast growth factor and fibroblast growth factor receptor protein expression in the formation of fibrotic focus in invasive ductal carcinoma of the breast. Jpn J Cancer Res 1997;88:877-85.

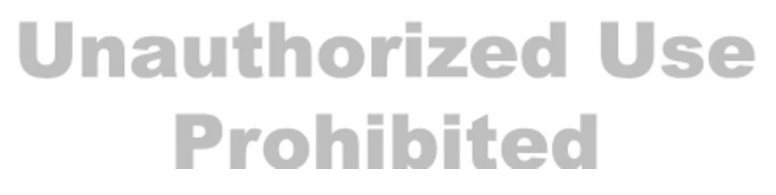

